\title{
THEOREMS ON STRONG RIESZ SUMMABILITY FACTORS
}

\author{
by B. L. R. SHAWYER \\ (Received 24th October 1964)
}

\section{Introduction}

Throughout this note it will be assumed that

(i) unless otherwise stated, $k$ is a positive integer,

(ii) $a \geqq 0 ; m>-1$,

(iii) $q \geqq 1 ; q^{\prime}$ is the conjugate number to $q$, and is defined by

$$
\frac{1}{q}+\frac{1}{q^{\prime}}=1
$$

(iv) the functions $\phi(w), \psi(w)$ are defined in $[0, \infty)$ with absolutely continuous $k$ th derivatives in every interval $[a, W]$,

(v) $\phi(w)$ is non-negative and unboundedly increasing,

(vi) $\lambda=\left\{\lambda_{n}\right\}$ is an unboundedly increasing sequence with $\lambda_{1}>0$.

Given an infinite series, $\sum_{n=1}^{\infty} a_{n}$, define

and write

$$
A_{m}(w)= \begin{cases}\sum_{\lambda_{n}<w}\left(w-\lambda_{n}\right)^{m} a_{n} & \text { if } w>\lambda_{1} \\ 0 & \text { otherwise }\end{cases}
$$

$$
A(w)=A_{0}(w) \text {. }
$$

If $w^{-m} A_{m}(w)$ tends to a finite limit, $S$, as $w \rightarrow \infty$, the series $\sum_{n=1}^{\infty} a_{n}$ is said to be summable $(R, \lambda, m)$ to sum $S$. If, in addition, $w^{-m} A_{m}(w)$ is of bounded variation with respect to $w$ in the range $[0, \infty)$, the series is said to be absolutely summable $(R, \lambda, m)$ to sum $S$, or summable $|R, \lambda, m|$ to sum $S$. Further, if there exists a number, $S$, such that

$$
\int_{0}^{w}\left|A_{m-1}(t)-S t^{m-1}\right|^{q} d t=o\left\{w^{(m-1) q+1}\right\},
$$

the series is said to be strongly summable $(R, \lambda, m)$ with index $q$ to sum $S$, or summable $[R, \lambda ; m, q]$ to sum $S$. Here it is assumed that $m q^{\prime}>1$. $\dagger$ Strong

$\dagger$ That $m q^{\prime}>1$ is essential is pointed out by Glatfeld in $\S 1$ of (8). However, he uses a different notation and definition. 
summability with index 1 is usually written as summability $[R, \lambda, m]$.

The object of this note is to obtain conditions sufficient for the truth of the proposition:

$$
\begin{aligned}
P: & \sum_{n=1}^{\infty} a_{n} \psi\left(\lambda_{n}\right) \text { is summable }[R, \phi(\lambda) ; k, q] \text { whenever } \\
& \sum_{n=1}^{\infty} a_{n} \text { is summable }[R, \lambda ; k, q] .
\end{aligned}
$$

I prove the following theorems:

Theorem 1. If there exists a function $\gamma(w)$, positive for $w \geqq a$, such that

(i) (a) $w^{n} \psi^{(n)}(w)=O\left[\left\{\frac{\gamma(w)}{w}\right\}^{k-n-1 / q^{\prime}}\right]$ for $n=0,1, \ldots, k-1$ and $w \geqq a$,

(b) $w^{k} \psi^{(k)}(w)=O(1)$ for $w \geqq a$,

(c) $\int_{a}^{\infty} t^{k}\left|\psi^{(k+1)}(t)\right| d t<\infty$,

(ii) (a) $\gamma(w) \phi^{\prime}(w)=O\{\phi(w)\}$ for $w \geqq a$, and if

(b) $\{\gamma(w)\}^{n-1} \phi^{(n)}(w)=O\left\{\phi^{\prime}(w)\right\}$ for $n=2,3, \ldots, k$ and $w \geqq a$,

(iii) there is a positive number, $N$, such that $\frac{\phi(w)}{w^{N}}$ is non-decreasing for $w \geqq a$, then $\boldsymbol{P}$.

Theorem 2. If

(i) $\phi(w)$ is a logarithmico-exponential function, $\dagger$

(ii) $\frac{1}{w} \preccurlyeq \frac{\phi^{\prime}(w)}{\phi(w)}$,

(iii) $\psi(w)=\left\{\frac{\phi(w)}{w \phi^{\prime}(w)}\right\}^{k-1 / q^{\prime}}, \ddagger$

then $\boldsymbol{P}$.

The following theorems are known [in theorems A and B, Srivastava does not restrict $k$ to integer values. In the case $0<k<1$, of theorem $\mathrm{B}$, additional restrictions are required]:

Theorem A. If

(i) $\phi(w)=e^{w}$,

(ii) $\psi(w)=w^{\left(-k+1 / q^{\prime}\right)}$,

then $P$.

$\dagger$ For definition of logarithmico-exponential functions, see (10).

$\ddagger k q^{\prime}>1$ since $k \geqq 1$ and $q^{\prime}>1$. 
Theorem B. If

(i) $\phi(w)$ is a logarithmico-exponential function,

(ii) $\phi(w)=O\left(w^{\delta}\right)$ for some $\delta>O$,

(iii) $\psi(w)=1$,

then $\boldsymbol{P}$.

Theorem C. If

(i) $\phi(w) \geqslant \phi^{\prime}(w) \geqslant \ldots \geqslant \phi^{(k)}(w)$,

(ii) $w^{-k} \phi(w)$ is monotonic increasing for $w \geqq a$,

(iii) $\psi(w)=w^{\left(-k+1 / q^{\prime}\right)}$,

then $\boldsymbol{P}$.

Theorem A, due to Srivastava (15), is a particular case of Theorem 2. Theorem B, a particular case of Theorem 1 , with $\gamma(w)=w$, is also due to Srivastava. For the proofs of the cases $q=1, q>1$, see (13), (14) respectively, Theorem $\mathrm{C}$, due to Hsiang (11), is included in Theorem 1, with $\gamma(w)=1$. $N=k$ and $\psi(w)=w^{-k+1 / q^{\prime}}$.

To complete the set of theorems analogous to those known for ordinary and absolute Riesz summability, we can deduce as a particular case of Theorem 1 :

Theorem D. If

(i) $\phi(w)=w$,

(ii) $\psi(w)$ is a logarithmico-exponential function tending to a finite limit, then $\boldsymbol{P}$.

Theorems similar to Theorem 1 exist for ordinary and absolute summability: for ordinary summability when $k$ is a positive integer, see Borwein (2); and when $k$ is any positive non-integral number, see Borwein and Shawyer (3): for absolute summability when $k$ is a positive integer, see Dikshit (6); and when $k$ is any positive non-integral number, see Dikshit (7), Borwein and Shawyer (4) and Ahmed (1).

Theorems analogous to Theorem 2 also exist for ordinary and absolute summability: for ordinary summability and all positive values of $k$ and for absolute summability when $k$ is a positive integer, see Guha (9); for absolute summability when $k$ is any positive non-integral number, see Borwein and Shawyer (4). The case, $q=1$, of Theorem 2 is known: see Borwein and Shawyer (5).

\section{Lemmas}

The following lemmas are required: 
Lemma 1. If there exists a number, $S$, such that

$$
\int_{0}^{w}\left|A_{k-1}(t)-S t^{k-1}\right|^{q} d t=o\left\{w^{(k-1) q+1}\right\}
$$

then $w^{-k} A_{k}(w) \rightarrow S$ as $w \rightarrow \infty$, This result is due to Srivastava (12).

Lemma 2. The $n$-th derivative of $\{f(t)\}^{m}$ is a sum of constant multiples of terms like

$$
\{f(t)\}^{m-\mu} \prod_{\nu=1}^{n}\left\{f^{(v)}(t)\right\}^{a_{\nu}}
$$

where $\alpha_{1}, \alpha_{2}, \ldots, \alpha_{n}$ are non-negative integers such that

$$
1 \leqq \sum_{v=1}^{n} \alpha_{v}=\mu \leqq \sum_{v=1}^{n} v \alpha_{v}=n
$$

Further, if $m$ is a positive integer, then $\mu \leqq m$.

This simple result is a special case of a theorem due to Faa di Bruno. See (16), pp. 88-89.

Lemma 3. If $\theta(t) \geqq 0, m>0$ and $m-n>0$, then the two assertions

and

$$
\int_{0}^{w} \theta(t) d t=o\left(w^{m}\right)
$$

$$
\int_{0}^{w} t^{-n} \theta(t) d t=o\left(w^{m-n}\right)
$$

are equivalent, it being assumed that both integrals converge at the origin.

Compare Lemma 2 in (8).

Lemma 4. If $\psi(w)$ is a logarithmico-exponential function satisfying $\psi(w) \preccurlyeq 1$, then, for any integer $k \geqq 1, \psi^{(k)}(w)<w^{-k}$ and

$$
\int_{a}^{\infty} t^{k} \psi^{(k+1)}(t) d t<\infty
$$

Proof. For $k=1, \psi^{\prime}(w)<w^{-1}$, since, otherwise $\psi^{\prime}(w) \geqslant w^{-1}$ which is in contradiction to the convergence of

$$
\int^{\infty} \psi^{\prime}(t) d t
$$

The truth of $\psi^{(k)}(t) \prec w^{-k}$ for $k \geqq 2$ follows from (10), $\S 5.22$.

Also, $\psi^{(k+1)}(t)$ is of constant sign for $t$ greater than some definite value, say $b$. Thus

$$
\int_{b}^{w} t^{k}\left|\psi^{(k+1)}(t)\right| d t= \pm \int_{b}^{w} t^{k} \psi^{(k+1)}(t) d t .
$$

By integrating by parts $k$ times, this is equal to a sum of a constant and constant multiples of

$$
w^{k} \psi^{(k)}(w), w^{k-1} \psi^{(k-1)}(w), \ldots, \psi(w)
$$


Since $\psi^{(k)}(w)<w^{-k}$, we deduce that

$$
\int_{b}^{w} t^{k}\left|\psi^{(k+1)}(t)\right| d t<\infty
$$

\section{Proof of Theorem 1}

Assuming without loss of generality that $S=0$, and that $A(w)=0$ for $0 \leqq w \leqq a$, from the hypotheses of the theorem and using Lemma 3 , we can immediately deduce that

$$
\int_{a}^{w} t^{-M}\left|A_{k-1}(t)\right|^{q} d t=o\left\{w^{(k-1) q+1-M}\right\}
$$

for $w \geqq a$, where $0 \leqq M<(k-1) q+1$.

It is sufficient for the proof of Theorem 1 , to show that there is a number, $\sigma$, such that, for $w \geqq a$,

where

$$
\int_{a}^{w} \phi^{\prime}(x)\left|F_{k-1}(x)-\sigma\{\phi(x)\}^{k-1}\right|^{q} d x=o\left(\{\phi(w)\}^{(k-1) q+1}\right)
$$

$$
F_{k}(x)=\int_{a}^{x}\{\phi(x)-\phi(t)\}^{k} \psi(t) d A(t)
$$

On integrating by parts, $F_{k-1}(x)$ can be expressed as a sum of constant multiples of

and

$$
I_{1}(x)=A_{k-1}(x)\left\{\phi^{\prime}(x)\right\}^{k-1} \psi(x)
$$

$$
I_{2}(x)=\int_{a}^{x} A_{k-1}(t)\left(\frac{\partial}{\partial t}\right)^{k}\{\phi(x)-\phi(t)\}^{k} \psi(t) d t .
$$

Consider first $I_{1}(x)$. In view of (3.1) † and conditions (i) (a), (ii) (b) and (iii) of Theorem 1 , we have that, for $w \geqq a$,

$$
\begin{aligned}
\int_{a}^{w} & \phi^{\prime}(x)\left|I_{1}(x)\right|^{q} d x \\
& =\int_{a}^{w}\left|A_{k-1}(x)\right|^{q}\left\{\phi^{\prime}(x)\right\}^{(k-1) q+1}|\psi(x)|^{q} d x \\
& =\int_{a}^{w} O\left[\left|A_{k-1}(x)\right|^{q} \frac{\{\phi(x)\}^{(k-1) q+1}}{\{\gamma(x)\}^{(k-1) q+1}}\left\{\frac{\gamma(x)}{x}\right\}^{q\left(k-1 / q^{\prime}\right)}\right] d x \\
& =\int_{a}^{w} O\left[x^{(N-1)((k-1) q+1\}}\left|A_{k-1}(x)\right|^{q}\left\{\frac{\phi(x)}{x^{N}}\right\}^{(k-1) q+1}\right] d x \\
& =O\left[\left\{\frac{\phi(w)}{w^{N}}\right\}^{(k-1) q+1} \int_{a}^{w} x^{(N-1)((k-1) q+1)}\left|A_{k-1}(x)\right|^{q} d x\right. \\
& =o\left[\{\phi(w)\}^{(k-1) q+1}\right],
\end{aligned}
$$

$\dagger$ Here, for (3.1), $M=(1-N)\{(k-1) q+1\}<(k-1) q+1$ since $N>0$. 
Consider next $I_{2}(x)$. Using Lemma 2 and Leibniz's theorem on the differentiation of a product, $I_{2}(x)$ can be expressed as a sum of constant multiples of integrals of the types

and

$$
I_{3}(x)=\int_{a}^{x} A_{k-1}(t)\{\phi(x)-\phi(t)\}^{k-1} \psi^{(k)}(t) d t
$$

$$
I_{m \mu}(x)=\int_{a}^{x} A_{k-1}(t) \psi^{(k-m)}(t)\{\phi(x)-\phi(t)\}^{k-1-\mu} \cdot \prod_{v=1}^{m}\left\{\phi^{(v)}(t)\right\}^{x_{v}} d t,
$$

where $\alpha_{1}, \alpha_{2}, \ldots, \alpha_{m}$ are non-negative integers such that

$$
\sum_{v=1}^{m} \alpha_{v}=\mu \leqq \sum_{v=1}^{m} v \alpha_{v}=m
$$

and $1 \leqq \mu \leqq k-1,1 \leqq m \leqq k$.

Consider first $I_{3}(x)$. Now

$$
k \int_{a}^{x} A_{k-1}(t) \psi^{(k)}(t) d t=A_{k}(x) \psi^{(k)}(x)-\int_{a}^{x} A_{k}(t) \psi^{(k+1)}(t) d t .
$$

Since $S=0$, in view of Lemma 1 , we have that

$$
A_{k}(x)=o\left(x^{k}\right) \text { for } x \geqq a \text {. }
$$

Hence, using this and condition (i) (b) of Theorem 1, we have that, for $x \geqq a$,

$$
A_{k}(x) \psi^{(k)}(x)=o\left(x^{k} \cdot x^{-k}\right)=o(1) .
$$

Further, in view of condition (i) (c) of Theorem 1, it follows that, for $x \geqq a$,

$$
\int_{a}^{x} A_{k-1}(t) \psi^{(k)}(t) d t=[\sigma+o(1)]
$$

and hence that, for $x \geqq a$,

$$
I_{3}(x)=[\sigma+o(1)]\{\phi(x)\}^{k-1},
$$

so there exists a number $\sigma$ such that, for $x \geqq a$,

$$
\int_{a}^{w}\left|I_{3}(x)-\sigma\{\phi(x)\}^{k-1}\right|^{q} \phi^{\prime}(x) d x=o\left[\{\phi(w)\}^{(k-1) q+1}\right] .
$$

Finally, consider $I_{m \mu}(x)$. First assume that $q>1$. Let $\theta$ be any number such that

$$
k-1 / q^{\prime}>\theta>k-1 / q^{\prime}-N .
$$

In view of the Holder inequality and conditions (i) (a), (ii) (a) and (b) and (iii) of Theorem 1 and (3.1), $\dagger$ it follows that, for $w \geqq a$,

$\dagger$ For (3.1), $M=\theta q<(k-1) q+1$. Also, $\mu q^{\prime}-1-\left\{(k-\theta) q^{\prime}-1\right\} / N>-1$ since $\theta>k-1 / q^{\prime}-N$. 


$$
\begin{aligned}
& \left|I_{m \mu}(x)\right|^{q}=\left|\int_{a}^{x} A_{k-1}(t) \psi^{(k-m)}(t)\{\phi(x)-\phi(t)\}^{k-1-\mu} \prod_{v=1}^{m}\left\{\phi^{(v)}(t)\right\}^{\alpha_{v}} d t\right|^{q}, \\
& \leqq \int_{a}^{x} t^{-\theta q}\left|A_{k-1}(t)\right|^{q}\{\phi(x)-\phi(t)\}^{(k-1-\mu) q} d t \\
& \cdot\left\{\int_{a}^{x}\left[\left|\psi^{(k-m)}(t)\right| t^{\theta} \prod_{v=1}^{m}\left|\phi^{(v)}(t)\right|^{\alpha_{v}}\right]^{q^{\prime}} d t\right\}^{q-1} \\
& \leqq\{\phi(x)\}^{(k-1-\mu) q} \int_{a}^{x} t^{-\theta q}\left|A_{k-1}(t)\right|^{q} d t \\
& \cdot\left\{\int_{a}^{x} O\left[t^{\theta q^{\prime}} \frac{\{\gamma(t)\}^{m q^{\prime}-1}}{t^{k q^{\prime}-1}} \frac{\left\{\phi^{\prime}(t)\right\}^{\mu q^{\prime}}}{\{\gamma(t)\}^{(m-\mu) q^{\prime}}} d t\right]^{q-1}\right\}, \\
& =o\left[\{\phi(x)\}^{(k-1-\mu) q} x^{(k-1-\theta) q+1} \cdot\left\{\int_{a}^{x} O\left[\frac{\{\phi(t)\}^{\mu q^{\prime}-1}}{t^{(k-\theta) q^{\prime}-1}} \phi^{\prime}(t)\right] d t\right\}^{q-1}\right] \text {, } \\
& =o\left[\{\phi(x)\}^{(k-1-\mu) q} x^{(k-1-\theta) q+1}\right. \\
& \left.\cdot\left(\int_{a}^{x} o\left[\left\{\frac{\phi(t)}{t^{N}}\right\}^{\left[(k-\theta) q^{\prime}-1\right] / N}\{\phi(t)\}^{\mu q^{\prime}-1-\left[(k-\theta) q^{\prime}-1\right] / N} \dot{\phi}^{\prime}(t)\right] d t\right)^{q-1}\right], \\
& =o\left[\{\phi(x)\}^{(k-1) q}\right]
\end{aligned}
$$

since $\mu \geqq 1$ and $k-1-\mu \geqq 0$.

Also, when $q=1$, in view of conditions (i) (a), (ii) (a) and (b) and (iii) of Theorem 1, and (3.1), $\dagger$ it follows that, for $w \geqq a$,

$$
\begin{aligned}
I_{m \mu}(x) & =\left|\int_{a}^{x} A_{k-1}(t)\{\phi(x)-\phi(t)\}^{k-1-\mu} \psi^{(k-m)}(t) \cdot \prod_{v=1}^{m}\left\{\phi^{(v)}(t)\right\}^{\alpha_{v}} d t\right|, \\
& =O\left[\{\phi(x)\}^{k-1-\mu} \int_{a}^{x}\left|A_{k-1}(t)\right| \frac{\{\gamma(t)\}^{m}}{t^{k}} \frac{\{\phi(t)\}^{\mu}}{\{\gamma(t)\}^{m}} d t\right], \\
& =O\left[\{\phi(x)\}^{k-1-\mu} \int_{a}^{x}\left|A_{k-1}(t)\right|\left\{t^{-N} \phi(t)\right\}^{\mu} t^{N \mu-k} d t\right], \\
& =o\left[\{\phi(x)\}^{k-1}\right]
\end{aligned}
$$

since $\mu \geqq 1$ and $k-1-\mu \geqq 0$.

Hence if follows that for $q \geqq 1$ and $w \geqq a$,

$$
\int_{a}^{w} \phi^{\prime}(x)\left|I_{m \mu}(x)\right|^{q} d x=o\left[\{\phi(w)\}^{(k-1) q+1}\right] .
$$

Thus, in view of the Minkowski inequality and (3.3), (3.4) and (3.5), there is a number, $\sigma$, such that (3.2) is true.

This completes the proof of Theorem 1 .

$\dagger$ For (3.1), $M=k-N \mu<k$. 


\section{Proof of Theorem 2}

We show that Theorem 2 is a particular case of Theorem 1. Define

$$
\gamma(w)=\frac{\phi(w)}{\phi^{\prime}(w)} .
$$

Condition (ii) (a) of Theorem 1 is now trivially true. In view of Lemma 1 (v) of (5)

whenever

$$
\phi^{(n)}(w) \preccurlyeq \phi(w)\left\{\frac{\phi^{\prime}(w)}{\phi(w)}\right\}^{n}
$$

$$
\frac{1}{w} \prec \frac{\phi^{\prime}(w)}{\phi(w)}
$$

That (4.1) is also true, when (4.2) is replaced by the weaker condition (ii) of Theorem 2, is given by the argument of (10), Theorem 24. (Here, this result is not explicitly stated, but the " $\preccurlyeq$ " is replaced by " (ii) (b) of Theorem 1 is satisfied.

Further, in view of (4.1), the argument in (5), Lemma 7, with index " $k$ " replaced by " $\left(k-1 / q^{\prime}\right)$ ", shows that condition (i) (a) of Theorem 1 holds, and that condition (i) of Theorem 2 is equivalent to condition (iii) of Theorem 1.

Finally, since the hypotheses imply that $\psi(w) \leqslant 1$, conditions (i) $(b)$ and (c) of Theorem 1 follow from the results of Lemma 4 .

This completes the proof of Theorem 2 .

\section{REFERENCES}

(1) Z. U. Afmed, On absolute Riesz summability factors, Rend. Circ. Math. Palermo, II, XI (1962), 91-104.

(2) D. Borwein, A theorem on Riesz summability, J. London Math. Soc. 31 (1956), 319-324.

(3) D. Borwein and B. L. R. Shawyer, On Riesz summability factors, Proc. Glasgow Math. Assoc. 5 (1962), 188-196.

(4) D. Borwern and B. L. R. Shawyer, On absolute Riesz summability factors, J. London Math. Soc. 39 (1964), 455-465.

(5) D. Borwern and B. L. R. Shawyer, On strong Riesz summability factors, London Math. Soc. 40 (1965), 111-126.

(6) G. D. Drkshit, On the absolute summability factors of infinite series, Indian J. Math. 1 (1958), 33-40.

(7) G. D. Dikshit, On the absolute summability factors of infinite series, II, Proc. Nat. Inst. Sci. India A 26 (1960), 86-94.

(8) M. Glatfeld, On strong Rieszian summability, Proc. Glasgow Math. Assoc. 3 (1957), 123-131. 
(9) U. C. GuHA, Convergence factors for Riesz summability, J. London Math. Soc. 31 (1956), 311-319.

(10) G. H. HARDY, Orders of Infinity (Cambridge Tract No. 12).

(11) Fu Cheng Hsiang, On the strong Riesz summability of an infinite series, Indian J. Math. 4 (1962), 47-52.

(12) P. Srivastava, On strong Rieszian summability of infinite series, Proc. Nat. Inst. Sci. India A 23 (1957), 58-71.

(13) P. Srivastava, On the second theorem of consistency for strong Riesz summability, Indian J. Math. 1 (1958), 1-16.

(14) P. Srivastava, On the second theorem of consistency for strong Riesz summability (II), Indian J. Math. 2 (1960), 97-117.

(15) P. SRIvastava, Theorems on strong Riesz summability, Quarterly J. Math. (2) 11 (1960), 229-240.

(16) C-J. De la Vallee Poussin, Cours d'analyse infinitesimale (Louvain, Paris).

THE UNIVERSITY

NotTINGHAM 